\title{
Using city-wide mobile noise assessments to estimate bicycle trip annual exposure to Black Carbon
}

\author{
Luc Dekoninck \\ Dick Botteldooren ${ }_{\mathrm{b}}$ \\ Information Technology, Acoustics Group, Ghent University \\ St-Pietersnieuwstraat 41, 9000 Ghent, Belgium ${ }^{\mathrm{a}, \mathrm{b}}$ \\ Luc Int Panis $\mathrm{s}_{\mathrm{d}, \mathrm{d})}$ \\ Flemish Institute for Technological Research (VITO), Boeretang 200, 2400 Mol, Belgiumc \\ Transportation Research Institute (IMOB), Hasselt University, Wetenschapspark 5 bus 6, 3590 \\ Diepenbeek, Belgium ${ }^{\mathrm{d}}$
}

\section{ABSTRACT}

Several studies have shown that a significant amount of daily air pollution exposure, in particular Black Carbon (BC), is inhaled during bicycle trips. Previously, the instantaneous BC exposure of cyclists was modeled as the sum of a background concentration and a local traffic related component based on a local assessment of traffic noise. We present a fast and low cost methodology to achieve a city-wide assessment of yearly average BC exposure of cyclists along their trips, based on a city-wide mobile noise sensing campaign.

The methodology requires participatory sensing measurements of noise, partially combined with $\mathrm{BC}$ and/or other air pollutants sensitive to local traffic variations. The combined measurements cover the spatial and meteorological variability and provide the data for an instantaneous exposure model. The mobile noise-only measurements map the full city; and yearly meteorology statistics are used to extrapolate the instantaneous exposure model to a yearly average map of in-traffic air pollution exposure. Less than four passages at each segment along the network with mobile noise equipment are necessary to reach a standard error of $500 \mathrm{ng} / \mathrm{m}^{3}$ for the yearly average $\mathrm{BC}$ exposure.

A strong seasonal effect due to the $\mathrm{BC}$ background concentration is detected. The background contributes only $25 \%$ to the total trip exposure during spring and summer. During winter the background component increases to $50-60 \%$. Engine related traffic noise along the bicyclist's route is a valid indicator of the $\mathrm{BC}$ exposure along the route, independent of the seasonal background. Low exposure route selection results in an exposure reduction of $35 \%$ in winter and $60 \%$ in summer, sensitive to the weather conditions, specific trip attributes and the available alternatives.

The methodology is relevant for further research into the local effects of air pollution on health. Mobile noise mapping adds local traffic data including traffic dynamics into the air pollution exposure assessments. Local policy makers and urban planners can use the results to support the implementation of low exposure infrastructure, create awareness through route planners and achieve behavioral changes toward active travel modes.
Highlights
- Mobile noise level to within $2 \mathrm{~dB}$ is sufficient to estimate annual $\mathrm{BC}$ exposure of cyclists
- Mapping mobile noise provides city-wide yearly averaged in-traffic air pollution exposure
- A low-cost methodology for city-wide evaluation of noise and air pollution is presented
- Cyclists can reduce exposure by 35-60\% through selecting low exposure routes

\footnotetext{
a) email: luc.dekoninck@intec.ugent.be (corresponding author, tel +32 926499 95)

b) email: dick.botteldooren@intec.ugent.be

c) email: luc.intpanis@vito.be; luc.intpanis@uhasselt.be
} 
Keywords: Black Carbon, Vehicle Noise, Personal exposure, Cyclists, Traffic, Active mobility

\section{Introduction}

Exposure to particulate matter is currently regulated in PM standards that only distinguish between the size of the particles but not between the composition and thus origin of the particulate matter. The soot fraction or Black Carbon (BC) is a fraction of the PM directly related to combustion processes. Recent evidence, summarized by the World Health Organization (WHO), documents the relevance of $\mathrm{BC}$ for evaluating traffic related health effects (WHO Europe, 2012). BC is more sensitive to traffic emissions and is able to detect local exposure differences not available in the $\mathrm{PM}_{10}$ evaluations. The first epidemiological results based on $\mathrm{BC}$ exposure detect health effects up to ten times stronger compared to the similar evaluations based on $\mathrm{PM}_{10}$ (Janssen et al. 2011).

Large personal exposure measurement campaigns prove the relevance of the in-traffic exposure contribution to daily personal exposure (Dons et al., 2011, 2012). Technology for mobile air pollution measurements is however scarce and expensive. The high variability of the in-traffic exposure is partially due to the strong influence of meteorology on the exposure, swamping the variability due to the local traffic densities and dynamics (Dons et al., 2013). In previous work of the authors, an instantaneous spatiotemporal model based on mobile noise measurements for cyclists was proposed (Dekoninck et al., 2013). It was shown that noise measurements are a good proxy for local traffic intensity and local traffic dynamics. The instantaneous spatiotemporal model splits the BC exposure of a cyclist into a background component and a component of local origin. In the Flemish region, only one continuous monitoring station was available at the time of the measurement campaign that can act as a background concentration (Antwerpen-Linkeroever 40AL01). After adjusting for the background contribution the local variation in the traffic density and traffic dynamics successfully predicts the BC exposure of the cyclists using four parameters: the low frequency noise $\left(\mathrm{L}_{\mathrm{OLF}}\right)$ related to the traffic volume and engine throttle, the difference between high and low frequencies $\left(\mathrm{L}_{\mathrm{HFmLF}}\right)$ relating to the traffic speed, the instantaneous wind speed and the street canyon index (StCan). Wind speed and street canyon features affect the dispersion of BC. The background adjusted model significantly reduced the temperature dependency of the bicyclist exposure. All temperature dependency, regardless of the origin (other BC sources, meteorology or vehicle fleet related aspects) is resolved in the background contribution. In other work, the instantaneous model approach was validated in completely different traffic 
conditions (Bangalore, India). The quality of the background adjustment was related to the properties of the background measurement location (Dekoninck et al, 2015). Meteorological conditions thus enter this model directly through the wind speed and indirectly via temperature and wind speed in the background $\mathrm{BC}$ concentration. Wind direction was not included in the model. The strongest component in the local traffic related exposure is the traffic within meters from the cyclists, resulting in correlation of 0.86 with only four parameters. Wind direction is not relevant at such small distances to the source. The noise measurements quantify the local traffic and traffic dynamics. The strengths of the method are based on the possibility to quantify the local traffic properties and by adding missing traffic data at low density traffic roads.

For many applications however, there is a strong interest in annual average rather than instantaneous levels of exposure. The internal validation of the instantaneous model -predicting $25 \%$ of the trips using models fit on the other $75 \%$ of the trips- resulted on average in a correlation of the trip averaged BC exposure of 0.77 (Dekoninck et al., 2013). The intrinsic quality of the instantaneous model to predict a single trip under any meteorological conditions or trajectory is strong and enables the simulation of yearly average exposure during bicycle trips without further validation. One of the remaining questions is whether subtracting a measured background concentration results in a valid local contribution that can actually be interpreted as the contribution of the local traffic $\left(\mathrm{BC}_{\mathrm{loc}}\right)$. If this is the case, the model should result in $\mathrm{BC}_{\text {loc }}$ approaching zero with low traffic intensity. As an alternative, the behavior of the strongest component in the spatiotemporal model, LoLF is proposed as a good indicator of the background adjustment since this component is directly related to the presence of traffic. When the spatiotemporal model reveals a linear relationship between LolF $_{\text {f }}$ and $\log \left(\mathrm{BC}_{\mathrm{loc}}\right)$, the background adjusted exposure will by consequence only include local traffic related exposure. For low LoLf, the local contribution in the instantaneous model dwindles to values close to the detection limit of the $\mathrm{BC}$ measurement equipment. Therefore the background location and the applied background correction are valid.

In each mobile measurement campaign only a limited number of potentially occurring situations is sampled. Bias is introduced into the measurements due to the combination of route choice, instantaneous meteorological conditions and instantaneous background exposure, each of them subject to unintended selection bias. It is therefore impossible to perform a measurement campaign covering all combinations of the exposure variables with unbiased exposure as the result. This paper will therefore introduce a methodology 
to convert a map of biased mobile measurements into a yearly average unbiased BC exposure map building upon the available instantaneous model (Dekoninck et al., 2013). This will be achieved in two phases. First the instantaneous exposure will be extrapolated to a yearly average by applying the spatiotemporal model for all meteorological situations to all available mobile noise measurements. In the second phase the instantaneous noise exposure is replaced by the average noise exposure at that location. In this way all dependence on meteorology can be removed, and the typical traffic situation at each location along the network is characterized using mobile noise measurements from a small but sufficient number of passages. This results in a spatial model, only based on local traffic related features (derived from noise) and a street canyon index (a parameter describing the accumulation of BC in narrow streets).

Several research questions will be addressed: how can a mobile noise measurement campaign be used to predict the annual average BC exposure for cyclists and how many noise measurement trips are necessary to reliably estimate the local traffic contribution. In addition, the effect of seasonal meteorological changes on the local traffic related BC exposure and on the total exposure will be illustrated. The potential exposure reduction by choosing low exposure routes is quantified.

\section{Methodology and data processing}

\subsection{Methodology}

The instantaneous model is based on 209 rush hour commuting trips by bicycle from the villages to the west of Ghent (Belgium) into the city center. More than $75 \mathrm{~km}$ of distinct roads were sampled at least 3 times. The BC measurements are performed with the micro-aethalometer AE51 (Aethlabs.com, San Francisco). The details are available in Dekoninck et al., 2013 and the supplementary data. The instantaneous logtransformed model will be referred to as $\mathrm{BC}_{\text {loc,temporal }}$ in this paper. The subscript 'temporal' is added to avoid confusion with the spatial yearly average models that will be developed in this work. Thus, to obtain a meteorology averaged BC exposure of cyclists, only wind speed and background concentration have to be accounted for. To implement a correction towards yearly representative meteorological conditions, the meteorological conditions are categorized according to the meteorological dependencies in $\mathrm{BC}_{\text {loc,temporal }}$ : wind speed and background concentration. A joint distribution over these two variables should be used because 
background $\mathrm{BC}$ concentration itself strongly depends on wind speed. The meteorological classes are presented in Section 2.2.

To obtain a yearly averaged value, the $\mathrm{BC}_{\text {loc,temporal }}$ model is applied for a sufficiently large number of bicycle commuting trips passing through each evaluation point along the road network for each combined meteorological - background concentration class observed during a year. This means that the instantaneous wind speed and background concentration of that specific trip are replaced in the model by the wind speed and background concentration of the evaluated meteorological class. For each spatial evaluation point, the resulting dataset contains a $\mathrm{BC}$ estimate for each noise-measuring trip and each meteo/background class, Met $_{c l}$. A weighted average is applied according to the frequency of occurrence during a year of Met $_{c l}$, merging the results spatially for all trips passing by at a specific location.

In mathematical form, the procedure is summarized as follows. The local contribution to the measured $\mathrm{BC}$ concentration $\mathrm{BC}_{\text {loc,meas,i,j }}$ for a location $i$ and during a trip $j$, is written as:

$$
B C_{\text {loc }, \text { meas }, i, j}=B C_{\text {tot }, \text { meas }, i, j}-B C_{b g, j}
$$

140 Using the previously derived model, this contribution is approximated using Generalized Additive Models (GAM) (Wood, 2006). Several authors in air pollution research have applied this technique successfully

142 (Dominici et al., 2002, Pearce et al., 2011, Li et al., 2013). The modeled BC concentration is obtained for each 143 location i during trip $\mathrm{j}$ as:

$$
\mathrm{BC}_{\text {loc,temporal,i,j }}=\exp \left(\operatorname{gamBC}_{\text {loc,temporal }}\left(\mathrm{L}_{\mathrm{OLF}, \mathrm{i}, \mathrm{j}}, \mathrm{L}_{\mathrm{HFmLF}, \mathrm{i}, \mathrm{j}}, \operatorname{StCan}_{\mathrm{i}}, \mathrm{WS}_{\mathrm{j}}\right)\right)
$$$$
\mathrm{BC}_{\text {tot,temporal,i,j }}=\exp \left(\text { gamBC }_{\text {loc,temporal }}\left(\mathrm{L}_{\mathrm{OLF}, \mathrm{i}, \mathrm{j}}, \mathrm{L}_{\mathrm{HFmLF}, \mathrm{i}, \mathrm{j}}, \operatorname{StCan}_{\mathrm{i}}, \mathrm{WS} \mathrm{S}_{\mathrm{j}}\right)\right)+\mathrm{BC}_{\mathrm{bg}, \mathrm{j}}
$$

Note that all spatiotemporal dependence of the GAM model is implicitly included via the model covariates and that the background contribution is assumed location independent. Applying the wind speed and $\mathrm{BC}$ background concentration for all meteorological conditions Met $_{c l}$ on each trip j:

$$
\mathrm{BC}_{\text {loc,year, }, \mathrm{ij}}=\frac{1}{\sum_{\text {Metcl }} \mathrm{w}_{\text {Metcl }}} \sum_{\text {Metcl }} \mathrm{w}_{\text {Metcl }}\left(\text { gamBC }_{\text {loc,temporal, }, \mathrm{i}, \mathrm{j}}\right)
$$

$$
\mathrm{BC}_{\text {tot,year,i,j }}=\frac{1}{\sum_{\text {Metcl }} \mathrm{w}_{\text {Metcl }}} \sum_{\text {Metcl }} \mathrm{w}_{\text {Metcl }}\left(\text { gamBC }_{\text {tot,temporal, }, \mathrm{j},}\right)
$$


151 Note that this result still has a weak dependence on trip number, and thus time, via the measured sound 152 parameters and via the chosen route at that specific time. By aggregating all measurement trips to a spatial 153 point $\mathrm{p}_{\mathrm{i}}$ along the network, the spatial distribution is obtained:

154

$$
\begin{aligned}
& \mathrm{BC}_{\text {loc,year,i }}=\frac{1}{\mathrm{n}} \sum_{\mathrm{j}} \mathrm{BC}_{\text {loc,year, } \mathrm{i}, \mathrm{j}} \\
& \mathrm{BC}_{\text {tot,year }, \mathrm{i}}=\frac{1}{\mathrm{n}} \sum_{\mathrm{j}} \mathrm{BC}_{\text {tot }, y e a r, \mathrm{i}, \mathrm{j}}
\end{aligned}
$$

Where for each spatial point $\mathrm{p}_{\mathrm{i}}$ the collection of trips $\mathrm{j}$ passing point $\mathrm{p}_{\mathrm{i}}$ contribute to the yearly average. In the last phase, the instantaneous noise levels are replaced by the average noise levels at each location $\mathrm{p}_{\mathrm{i}}$. (eq. 7 and 8). All meteorological variability is included in the input data (eq. 5 and 6) and no instantaneous meteorological covariates are necessary in the yearly meteorology based GAM models (eq. 9 and 10):

$$
\mathrm{L}_{\mathrm{OLF}, \mathrm{i}, \mathrm{map}}=\frac{1}{\mathrm{n}} \sum_{\mathrm{j}} \mathrm{L}_{\mathrm{OLF}, \mathrm{i}, \mathrm{j}}
$$

$$
\operatorname{gamBC}_{\text {tot,year }} \equiv \log \left(\mathrm{BC}_{\mathrm{tot}, \mathrm{year}, \mathrm{i}}\right)=\operatorname{gam}\left(L_{O L F, i, \text { map }}, L_{H F m L F, i, m a p}, \operatorname{StCan}_{i}\right)
$$

This results in a function that converts the average mobile noise measurement map to an average $\mathrm{BC}$ map, independently of the trip sampling paths. In Figure 1, the spatial variability of the two noise covariates ( $\mathrm{L}_{\mathrm{OLF}, \mathrm{i}, \mathrm{map}}$ and $\mathrm{L}_{\mathrm{HFmLF}, \mathrm{i}, \mathrm{map}}$ ) and the street canyon index $\mathrm{StCan}_{\mathrm{i}}$ are illustrated, as well as the background adjusted local component of the $\mathrm{BC}$ measurements $\left(\mathrm{BC}_{\mathrm{loc}, \mathrm{meas}, \mathrm{i}, \mathrm{map}}\right)$ spatially averaged in a similar way as the noise covariates (eq. 7 and 8). Local BC contributions are highly variable and are affected by local traffic variability. 

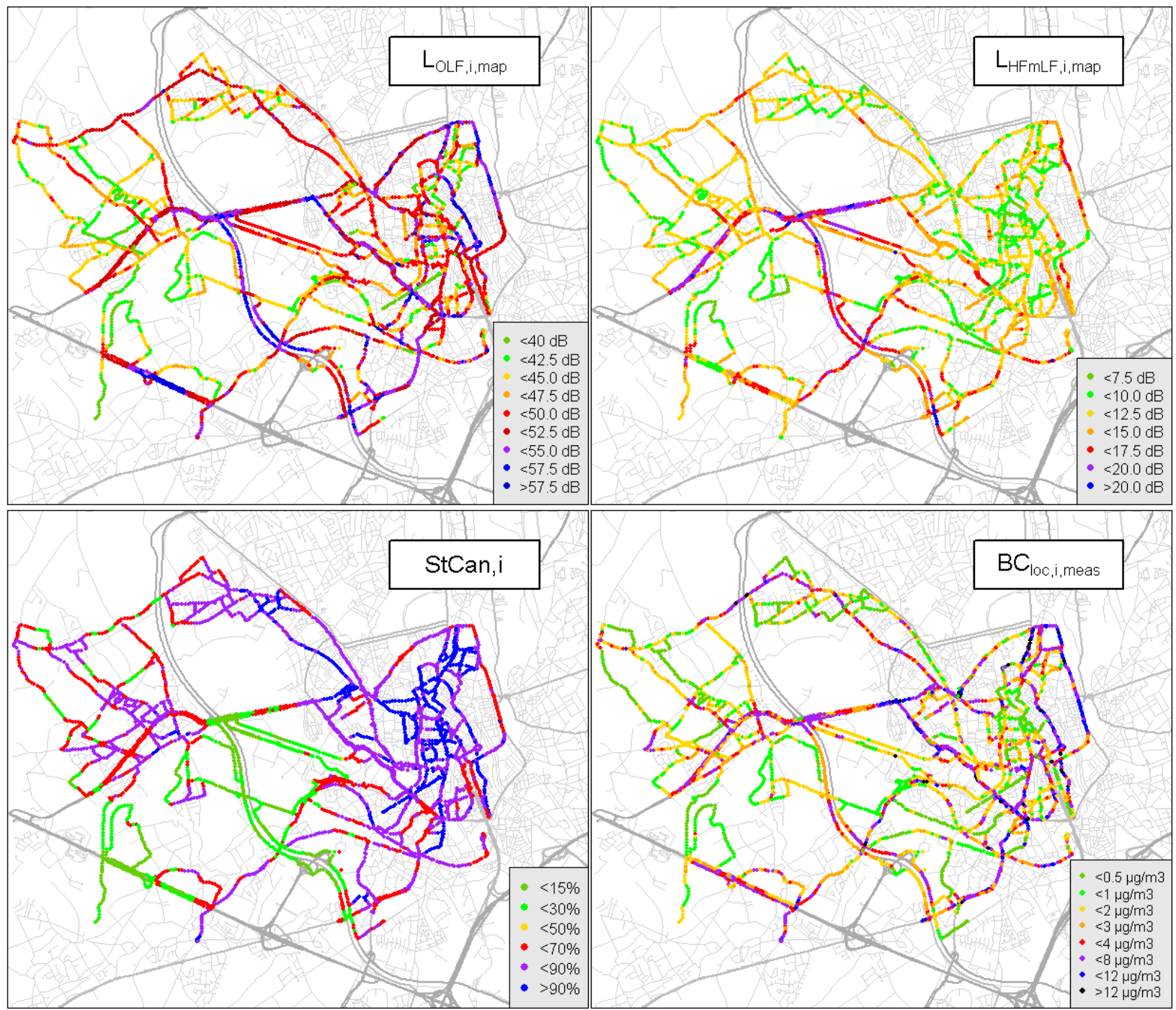

Figure 1: Three maps showing the spatial covariates (LoLf,i,map, $\mathrm{L}_{\mathrm{HFmLF}, \mathrm{i}, \mathrm{map}}$ and $\mathrm{StCan}_{\mathrm{i}}$ ). The fourth map shows the average local contribution to the $\mathrm{BC}$ exposure measurements: $\mathrm{BC}_{\text {loc,meas,i,map. }}$

\subsection{Meteorology classes}

The combined mobile noise and BC measurement campaign stretched from November 2010 till background adjustment in the spatiotemporal model. Further information is available at http://www.irceline.be. 

in the statistics, since the model is only based on rush hour measurements. For the year 2011 this resulted in 160 distinct meteorological / background concentration classes (referred to as $\mathrm{Met}_{\mathrm{cl}}$ ), each class occurring in the year 2011 with a weight wMet $_{c l, 2011}$. The wind speed classes ranged from 0 to $11 \mathrm{~m} / \mathrm{s}$ and the BC background concentrations from 0 to $16 \mu \mathrm{g} / \mathrm{m}^{3}$. The distribution of the wind speed classes, $\mathrm{BC}$ background classes and the combined occurrences are presented in Figure 2. The size of the circles is proportional to the occurrence $\mathrm{wMet}_{\mathrm{cl}, 2011}$ of the meteorological situations. The sparse BC background values above $7 \mu \mathrm{g} / \mathrm{m}^{3}$ were added to the $7 \mu \mathrm{g} / \mathrm{m}^{3}$ class for easy presentation in the plot only.
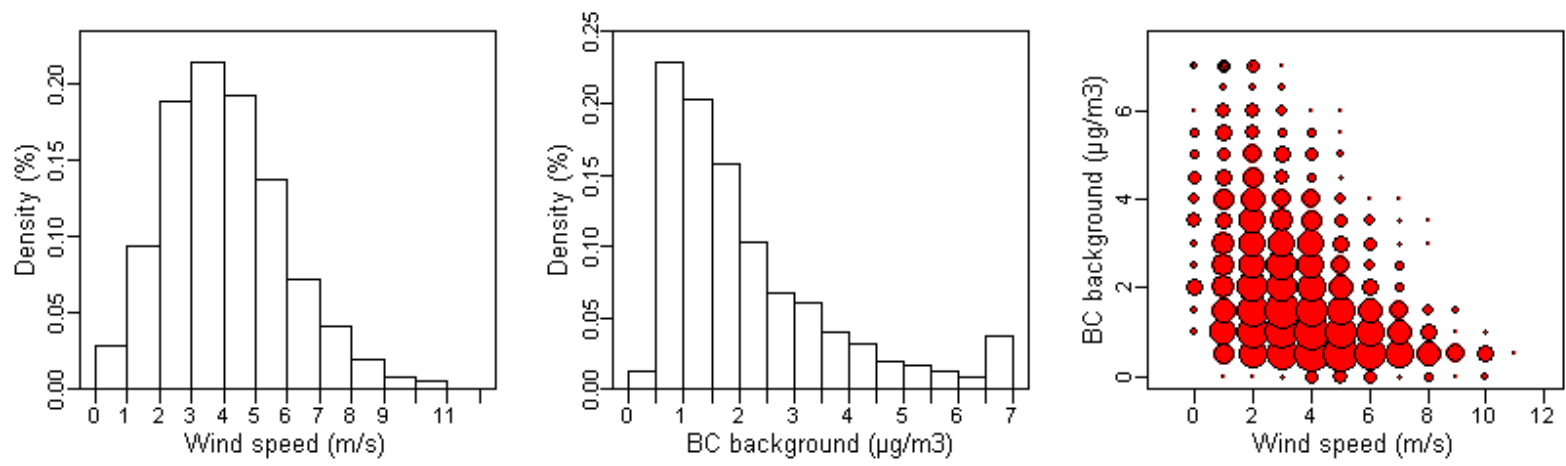

191

192

193

194

195

Figure 2: Histograms of the wind speed (A), BC background exposure (B) and the combined occurrence of wind speed and background exposure (based on hourly wind speed data in Melle and 30 minute BC data for station 40ALO1 obtained respectively from VMM and KMI).

\section{Results}

\subsection{A model for yearly averaged BC concentration}

The data processing is based on data from Dekoninck et al, 2013, referred to as BDS. After applying Eq.(3) and (4) on BDS the yearly meteorology adjusted $\mathrm{BC}$ exposure is available as $\mathrm{BC}_{\text {year,loc,i,j }}$ and $\mathrm{BC}_{\text {year,tot,i,j }}$ for each location $\mathrm{i}$ and each trip j. The new datasets are representative for the yearly averaged meteorological condition, removing the bias due to the not representative sample of meteorology of the trips that passed at

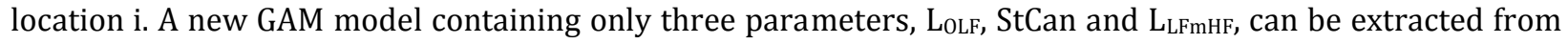
the new dataset, since the variability due to the wind speed is removed. The resulting models, gamBC $\mathrm{loc}_{\text {,year }}$ and the gamBC $\mathrm{Coty}_{\text {year }}$ are presented in Table 1 . The GAM models are almost trivial and virtually perfect, since they are based on the output of the $\mathrm{BC}_{\text {loc,temporal }} \mathrm{GAM}$ model. The only variability left in these GAM models is 
the difference in the instantaneous noise evaluation covariates $\mathrm{L}_{\mathrm{OLF}}$ and $\mathrm{L}_{\mathrm{HFmLF}}$ for the trips contributing to the location i.

\begin{tabular}{|c|c|c|c|c|c|c|c|}
\hline Model & Intercept & LoLF,i,map & 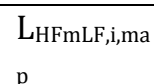 & StCan & $\begin{array}{l}\text { Deviance } \\
\text { explained }\end{array}$ & $\begin{array}{l}\text { Number } \\
\text { of points }\end{array}$ & AIC \\
\hline & & \multicolumn{3}{|c|}{ F-value } & $\%$ & $\mathrm{~N}$ & \\
\hline gamBC $_{\text {loc year }}$ & $8.11\left(3.3 \mu \mathrm{g} / \mathrm{m}^{3}\right)$ & 37427 & 10272 & 18874 & 99.5 & 3827 & -17064 \\
\hline gamBC $C_{\text {totyear }}$ & $8.60\left(5.4 \mu \mathrm{g} / \mathrm{m}^{3}\right)$ & 17766 & 4395 & 8046 & 98.9 & 3827 & -17980 \\
\hline
\end{tabular}

Table 1: Model results comparison, showing intercept, F-values, deviance explained and AIC.

The gamBC $C_{\text {loc,year }}$ model is the strongest model. The engine related noise covariate $\mathrm{L}_{\mathrm{oLF}}$ is the strongest are shown for gamBC $C_{\text {loc,year }}$ and gamBC $\mathrm{C}_{\text {totyear. }}$ In gamBC $\mathrm{Cot}_{\text {,year }}$ the low traffic condition (small $\mathrm{L}_{\mathrm{OLF}}$ ) converges to an lower limit, related to the yearly average $\mathrm{BC}$ background concentration. In the $\mathrm{L}_{\mathrm{OLF}}$ covariate of 212 gamBC loc,year a very linear relation is found, even for low values. Traffic evaluation through noise assessment 213 has a perfect linear log-log relationship with the engine related noise after adjusting for the yearly 214 meteorology distribution. The speed related covariate $\mathrm{L}_{\mathrm{HFmLF}}$ converges to a maximum. In situations with high 215 traffic speed the BC exposure does not increase any further for equal $\mathrm{L}_{\mathrm{OLF}}$. In the street canyon index a step216 like solution is visible. Below 0.5 the location is in rather open area, above 0.5 , dispersion is significantly 217 reduced.
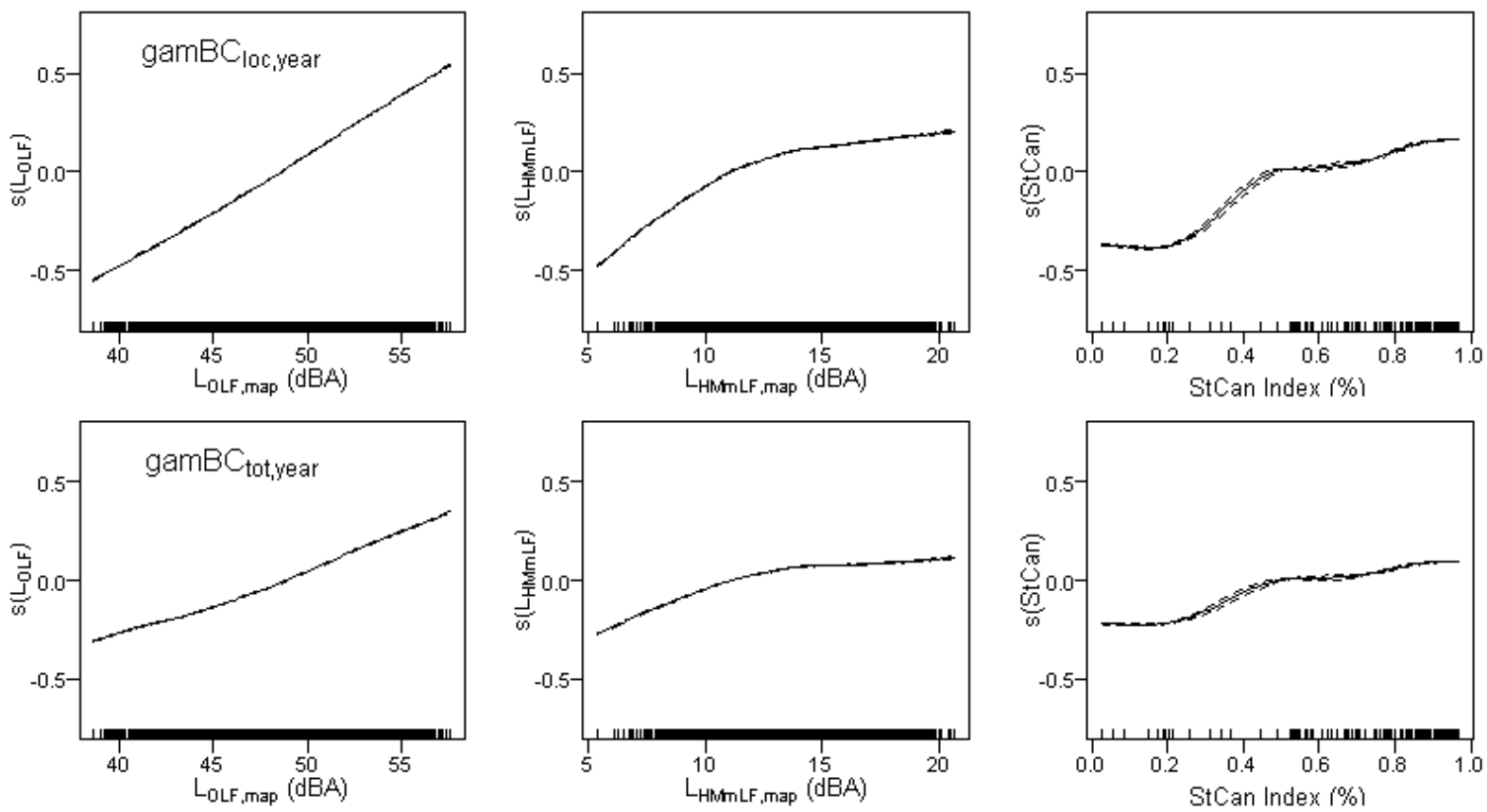
Figure 3: Splines of three covariates of the gamBC $\mathrm{Coc}_{\text {lyear }}\left(\right.$ top) and gamBC $\mathrm{Cot}_{\text {,year }}$ (bottom) models show strong linear behavior between the $\mathrm{OLF}$ and $\log (\mathrm{BC})$. In the total exposure the effect of the background exposure emerges, limiting the decrease of the exposure for low OLF.

Now we illustrate the effect of the different steps in modeling the yearly meteorology adjusted BC exposure using mobile noise data. A sequence of evaluations in Figure 4 illustrates the consecutive adjustments of the meteorology related effects on the bicyclist's exposure. The first evaluation (plot A) shows $\mathrm{BC}_{\text {tot,meas,j, }}$, the averaged measured $\mathrm{BC}$ exposure by trip (each dot is one trip) as a function of the strongest covariate $\mathrm{L}_{\mathrm{OLF}, \text { meas,j, }}$, the averaged measured noise level $\mathrm{L}_{\mathrm{OLF}}$ along trip j. The correlation is low. After adjusting the exposure for the background contribution $\left(\mathrm{BC}_{\text {loc,meas, } \mathrm{j}}\right)$ in plot $\mathrm{B}$ the correlation increases, but the wind speed is still an important variable in the personal exposure (see Dekoninck et al, 2013). The linear fit of the previous plot is repeated in the dashed line as a reference for each of the consecutive steps. In plot $\mathrm{C}$ the exposure is extrapolated to the yearly average meteorological conditions $\mathrm{BC}_{\text {loc,year,j }}$ for each trip $\mathrm{j}$. The correlation is enhanced up to 0.88 expressing the removal of the meteorology induced bias in the mobile measurement campaign. Several of the extremely high exposure trips are drastically reduced by applying the yearly average meteorology. The $y$-axis is adjusted to the new range. Plot D investigates the effect of replacing the actual noise measured along the trip by the average noise level for all passages at each location i passed by trip j, thus evaluating the trips according to the average mobile noise map for both noise covariates LoLF,i,map and $\mathrm{L}_{\mathrm{HFmLF}, \mathrm{i}, \mathrm{map}}$. Only a small change is detected for shifting from instantaneous noise level to the average over multiple passages, supporting the validity of replacing the instantaneous noise levels by the average noise levels. The correlation increases due to the removal of differences in the noise evaluation for trips passing at the same location i. Plot $\mathrm{E}$ replaces $\mathrm{BC}_{\text {loc,year,j }}$ by $\mathrm{BC}_{\text {tot,year,j. }}$. The correlation is not affected since the difference is only related to the change in background exposure. The yearly average background adjustment is added.
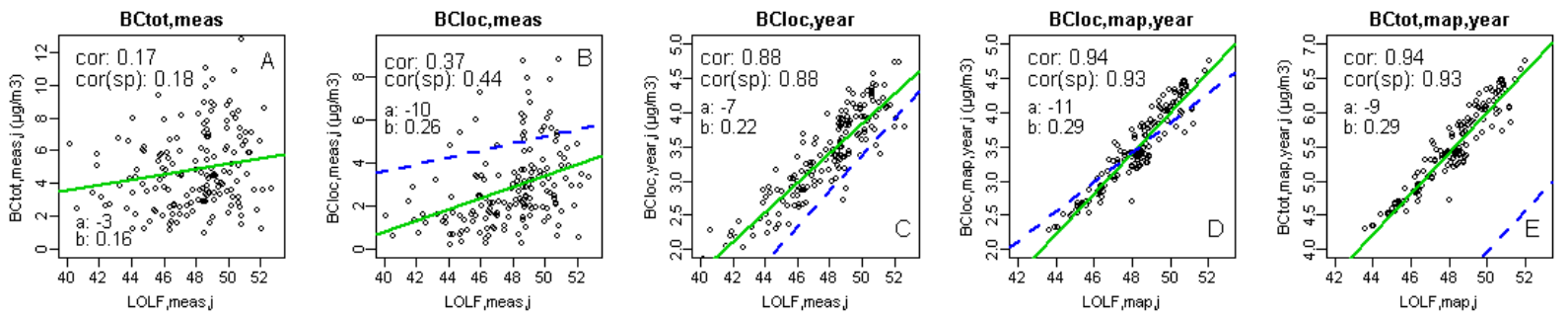

Figure 4: Visual representation of the sequential improvements of mobile measurements as a function of the trip average engine noise $\mathrm{L}_{\mathrm{OLF}}$ : average measured $\mathrm{BC}_{\text {tot,meas,j }}(\mathrm{A})$ along the trip and different exposure modeling steps: $\mathrm{BC}_{\text {loc,meas,j, }} \mathrm{BC}_{\text {loc,year,j }} \mathrm{BC}_{\text {loc,year,map, } \mathrm{j}}$ and $\mathrm{BC}$ tot,year,map,j.(B, C, D, E). Each 
plot includes a linear fit on the trip evaluation (green). The linear fit of the previous plot is repeated in dashed blue on the next plot. Note the changes in the $x$ and $y$-axis in the different steps.

The spatial result of the gamBC $C_{\text {loc,year }}$ and gamBC $C_{\text {tot,year }}$ is presented in Figure 5 . These maps are the yearly meteorological adjusted version of $\mathrm{BC}_{\text {loc,meas,i }}$ as presented in Figure 1 . The maps are much smoother due to the removal of meteo-related variability. They illustrate the sensitivity of the exposure to the local amount of traffic, traffic dynamics and the distance of the bicyclist to the local traffic. The difference between $\mathrm{BC}_{\text {loc,year }}$ and $\mathrm{BC}_{\text {totyear }}$ is a constant, the yearly averaged background adjustment.

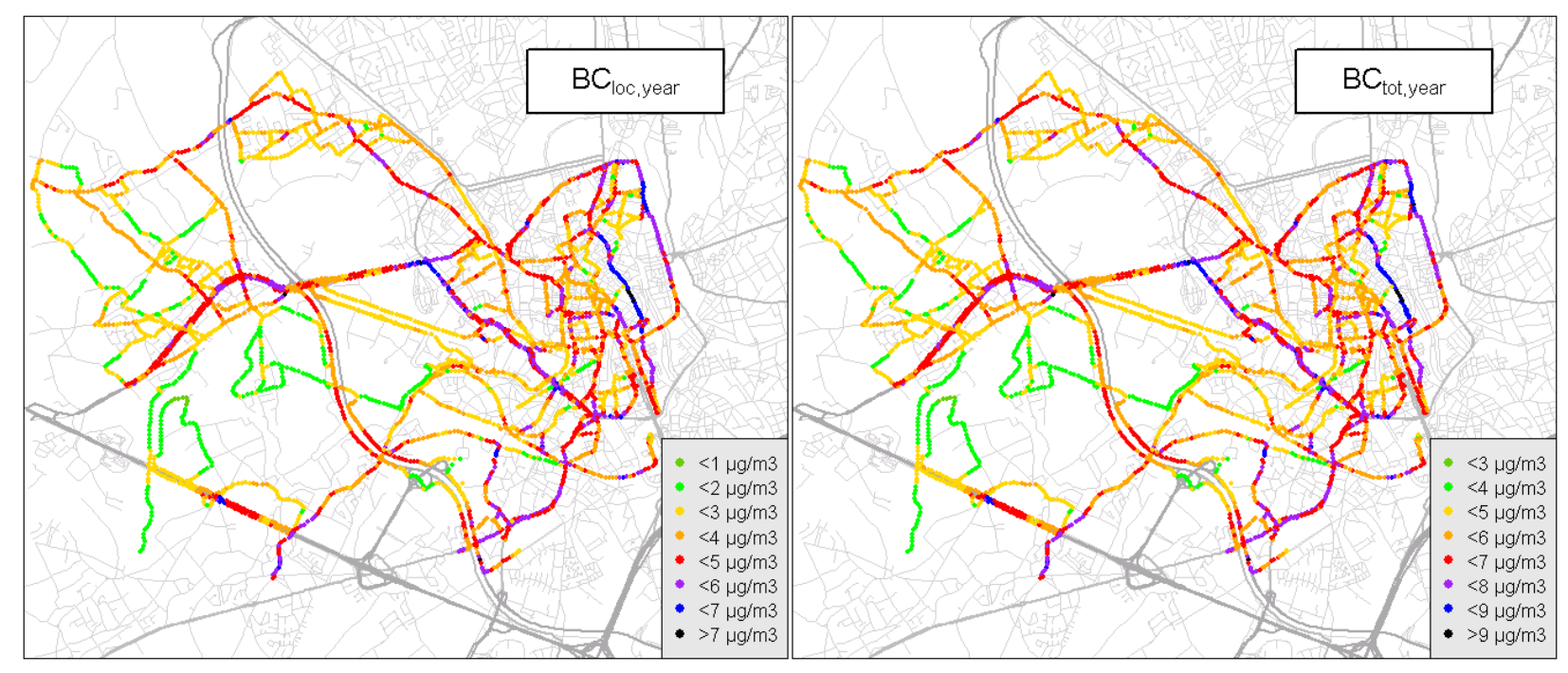

Figure 5: Visual representation of the yearly meteorology adjusted and noise mapped exposure based $\mathrm{BC}$ exposure for cyclists: local component $\mathrm{BC}_{\text {loc,year }}($ left) and total exposure $\mathrm{BC}$ tot,year (right).

\subsection{Number of noise measurements necessary for yearly averaged $\mathrm{BC}$ prediction}

Since bicyclist's BC exposure is highly sensitive to the meteorology, it is virtually impossible to achieve a representative sample of measurements for all possible combinations of local traffic and meteorological situations at a given location. Since the noise exposure at location $\mathrm{i}$ is not sensitive to the $\mathrm{BC}$ background concentrations and much less sensitive to wind speed and wind direction compared to BC exposure, it is expected that much less measurement repetition will be needed to quantify the local noise exposure at location i compared to $\mathrm{BC}$ exposure. Hence, the measurement error on the yearly averaged $\mathrm{BC}$ exposure is also expected to drop considerably with increasing number of trips. To confirm this expectation, the measurement error (standard deviation over all trips divided by the square root of the number of trips that contribute to the average) is calculated for each location $i$ with two or more passages. Figure 6 shows box plot 
statistics over all locations of this standard error for six parameters: $\mathrm{L}_{\mathrm{OLF}, \mathrm{i}}, \mathrm{L}_{\mathrm{HFmLF}, \mathrm{i}}, \mathrm{BC}_{\text {totmeas,i, }} \mathrm{BC}_{\text {loc,meas,i, }}$ $\mathrm{BC}_{\text {tot,year,i }}$ and $\mathrm{BC}_{\text {loc,year,i }}$ as a function of the number of passages $n$. For $\mathrm{BC}_{\text {tot,meas,i }}$ the standard error is not significantly improving with increasing number of passages if the number of passages is below 10, illustrating the high meteorology induced variability. That conclusion is also valid for $\mathrm{BC}_{\text {loc,meas, }}$, which is surprising since a large portion of the meteorological effects is already removed from the dataset by adjusting for instantaneous background exposure. For the yearly meteorology adjusted $\mathrm{BC}_{\text {totyear,i }}$ the standard error is reduced dramatically. With as little as four passages, the Q3 of the standard error of $\mathrm{BC}_{\text {loc,year, }}$ is smaller than $0.5 . \mu \mathrm{g} / \mathrm{m}^{3}$. In this dataset $95 \%$ of all locations have a standard error for LoLf below $2.0 \mathrm{~dB}$ and result in a standard error below $0.5 \mu \mathrm{g} / \mathrm{m}^{3}$ for $\mathrm{BC}_{\text {loc,year,i. }}$ For $\mathrm{BC}_{\text {tot,year, },}$ four passages result in a $\mathrm{Q} 3$ smaller than 0.7 . $\mu \mathrm{g} / \mathrm{m}^{3}$ and $90 \%$ of all locations in this dataset have a standard error for LoLf below $2.0 \mathrm{~dB}$ and result in a standard error below $0.75 \mu \mathrm{g} / \mathrm{m}^{3}$. Evaluating LoLF within $2 \mathrm{~dB}$ accuracy is more than sufficient to characterize the yearly averaged traffic related $\mathrm{BC}$ exposure.
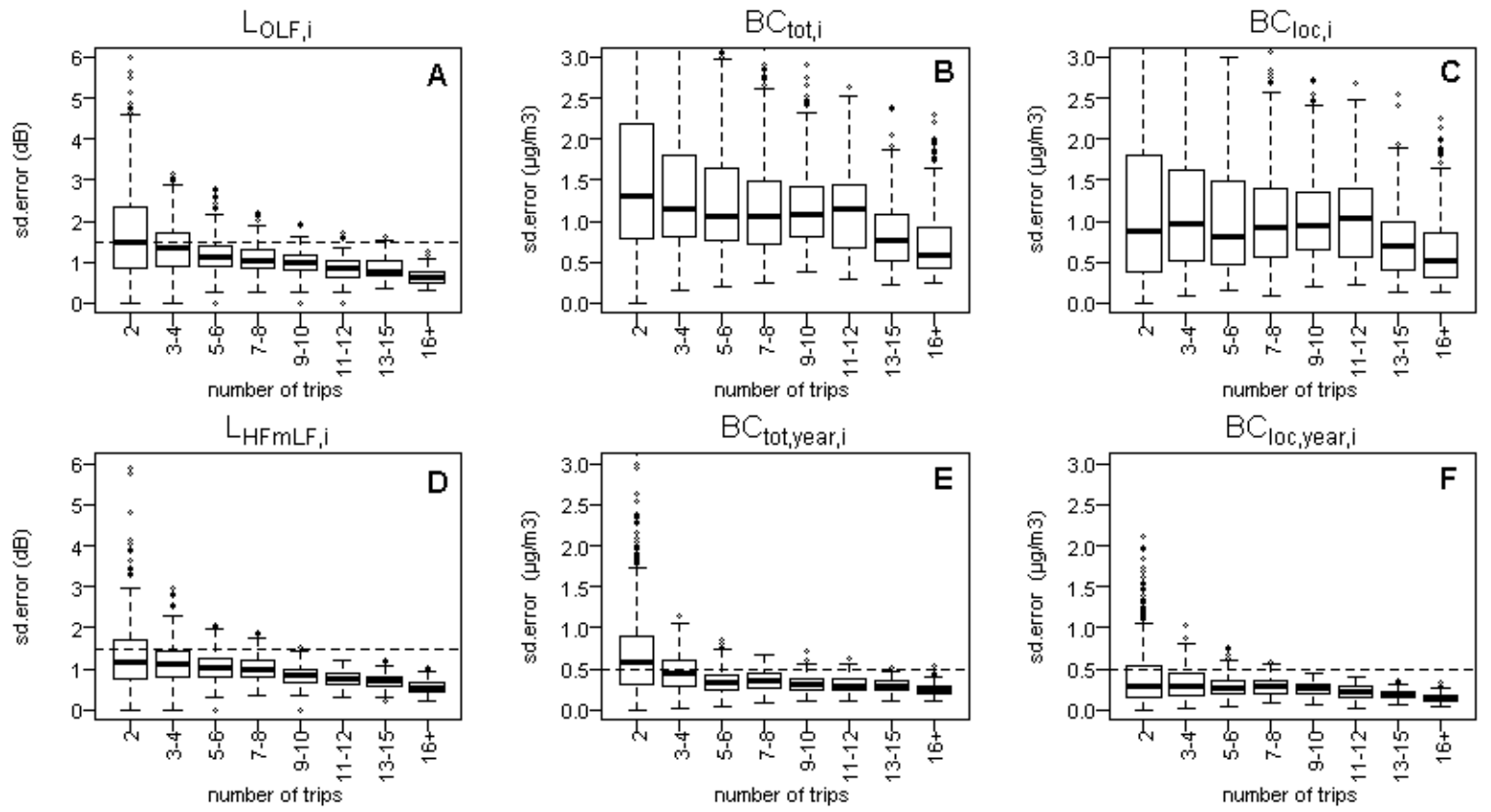


\subsection{Monthly variation in BC exposure}

In many countries the actual use of bicycles and the time spent outdoors depends on weather conditions and season. Hence, to correctly assess the yearly averaged exposure as well as to give advice to reduce personal exposure, one may need to account for the annual variation in meteorological conditions. In Figure $7 \mathrm{~A}$ the evaluation over all trips is presented, while restricting the meteorological weighting to the occurrence of the meteorological conditions for that specific month: $\mathrm{BC}_{\text {loc,month, }}$ and $\mathrm{BC}_{\text {tot,month,j. }}$. The distributions indicate the range of the high to low exposure trips. The corresponding monthly meteorological statistics for the wind speed and background concentrations are shown in Figure 7B. Note the atypical conditions in December 2011 with high wind speeds and low background exposure. The variability of the wind speed by month does not show a strong seasonal pattern, and since this is the only meteorological covariate in the GAM model for

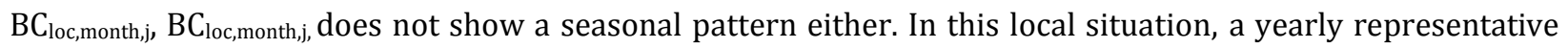
sample of wind speeds occurs each month. A strong seasonal effect on the $\mathrm{BC}_{\text {tot,year,j }}$ is visible, expressing the strong seasonal effect of the background exposure. In Figure 7C the relative contribution of the background exposure to the local traffic component is shown. In summer, the local contribution is almost $75 \%$ of the total contribution, in winter it drops to $50 \%$. For the larger part of the year, the local contribution is more than $60 \%$ of the total exposure. 

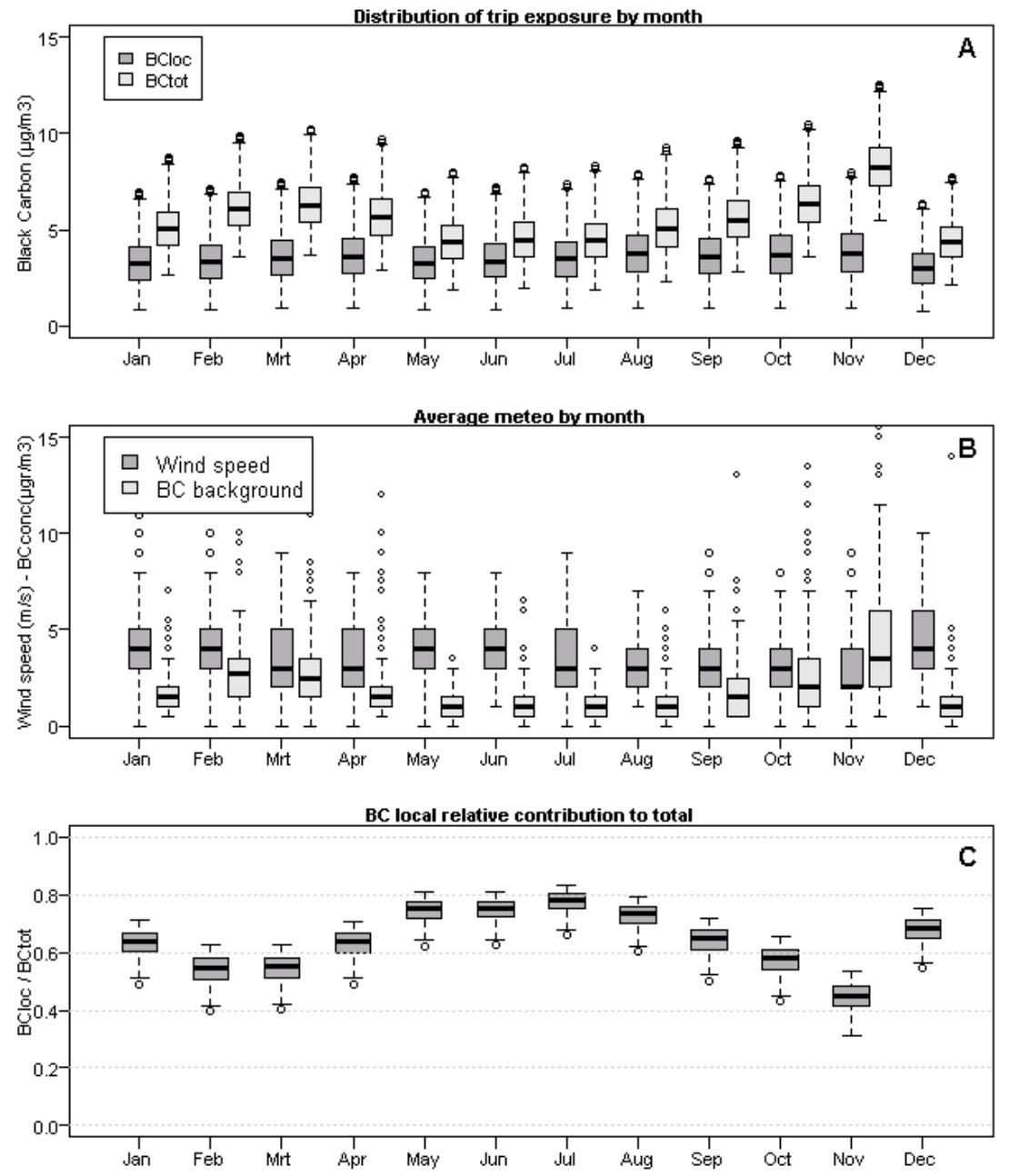

Figure 7: Monthly variation of the distribution of the local and total BC exposure by trip (A). Background $\mathrm{BC}$ and Wind speed show the effect of changing meteorological conditions over the year (B). Relative contribution of the local contribution to the total exposure (C).

\subsection{City-wide mapping and low exposure routes}

The proposed methodology for data collection uses mobile measurements on bicycle and consists of two simultaneous actions: during a limited set of trips $\mathrm{BC}$ and noise are measured jointly, preferably covering the full range of meteorological conditions and traffic conditions. These data are used to tune the coefficients in a local version of the $\mathrm{BC}_{\text {loc,temporal }}$ model to the specific emission of the local vehicle fleet, meteorological conditions and background concentrations. The $\mathrm{BC}_{\text {loc,temporal }}$ model is converted to the yearly meteorology adjusted GAM models as described in 2.1. An extensive mobile noise only measurement campaign can be performed to obtain spatial detail for a more extended area. In a numerical example: a city-wide participatory campaign with ten cheap noise measurement units, used by one-hundred participants for two weeks each can be completed in half a year. With a typical bicycle route of $10 \mathrm{~km}$, each measuring their standard route and a 
set of alternatives, will result in approximately 100 persons x 20 trips x $10 \mathrm{~km}$ or 20,000 km of mobile noise data. If the participants' origins and destinations are well distributed over the investigated city and suburbs, the majority of roads will be sampled adequately. The mobile noise measurements result in city-wide noise maps LoLF,i,map and LHFmLF,imap. Mapping any bicycle trajectory to the mobile noise maps (within the extent of the map) and applying the $\mathrm{BC}_{\text {loc,temporal }}$ results in an instantaneous meteorological sensitive estimate of the $\mathrm{BC}$ trip exposure. Applying the yearly or monthly meteorology adjusted models results in yearly or monthly averaged $\mathrm{BC}$ exposure, according to the requirements of the application.

To estimate the potential exposure improvement of alternative route choice, we take a closer look at the trips used for deriving the models presented in this article (Figure 4). Figures 4D and 4E show that trips performed during rush hour result in differences in $\mathrm{L}_{\mathrm{OLF}}$ of up to $10 \mathrm{~dB}$ with a matching reduction of over $50 \%$ in $\mathrm{BC}_{\text {loc,year }}$ and $35 \%$ in $\mathrm{BC}_{\text {totyear. }} \mathrm{A}$ reference trip with an average $\mathrm{LoLF}$,map,trip of $50 \mathrm{~dB}$ and an alternative trip of $46 \mathrm{~dB}$ will result in a decrease of $1.3 \mu \mathrm{g} / \mathrm{m} 3$, a reduction of $33 \%$ for the local traffic component and $22 \%$ on the total exposure in a far from extreme trip change scenario. In interpreting the results one should take into account that the travelled routes in this dataset were not optimized to detect the low exposure routes. The travelled routes were by design always switching from high to low exposure road segments to capture as much variability in traffic for similar meteorological conditions within a single trip. A systematic choice for a low exposure route for commuting could easily result in a 35 to $60 \%$ reduction in personal exposure depending on the season, but this also depends on the specific commute and the available alternatives for that trip.

\section{DISCUSSION}

\subsection{Predictive quality of the methodology}

At first sight the GAM models deviance explained is extremely high. When considering the procedure and the importance of the wind speed covariate in the spatiotemporal model, this is not surprising. All remaining variability that is not explained by the spatiotemporal model is -by design- not available in the yearly extrapolation. More important than the fact that the $\mathrm{gamBC}_{\text {loc,year }}$ and gamBC $\mathrm{Btotyear}_{\text {models }}$ are virtually perfect, is that all locations are included, even those that were sampled only once or twice, without affecting the quality of the GAM model. It was expected that the dataset should be restricted to a minimum number of 
passages to build a valid GAM model, but this was clearly not necessary. The quality of the model in the locations with low sampling is also visible in Figures $6 \mathrm{E}$ and $6 \mathrm{~F} .75 \%$ of the points with only two passages reach a yearly standard error below $500 \mathrm{ngr} / \mathrm{m}^{3}$ for the gamBC loc,year and almost $50 \%$ reach this value for the

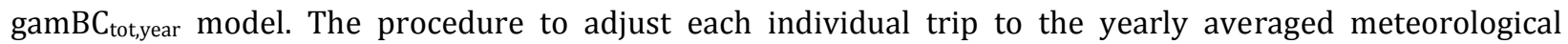
situation is effective. Dekoninck et al. (2012) concluded that an estimate of $\mathrm{L}_{\mathrm{OLF}}$ resulted in a standard error of approximately $1.5 \mathrm{~dB}$ after ten samples at a specific location in similar traffic conditions. The results in this paper show that a standard error of $1.5 \mathrm{~dB}$ does not have to be achieved to successfully apply this methodology. A standard error of $2.0 \mathrm{~dB}$ is more than sufficient to result in a valid prediction of the yearly $\mathrm{BC}$ exposure, which is achieved with four passages for a specific traffic condition. The data showed that it is extremely difficult to achieve a valid averaged $\mathrm{BC}$ exposure by increasing the number of passages without using the proposed modeling approach, as illustrated by the low reduction by number of trips in Figures $6 \mathrm{~B}$ and 6C. Noise measurements adequately quantify the local traffic situation and enable the disaggregation of the variability in the $\mathrm{BC}$ measurements into local traffic related and meteorology related contributions. This is valid for the instantaneous model and the yearly meteorology extrapolated model.

\subsection{Comparison with other mobile measurements}

A general review by Bigazzi and Figliozzi (2014) summarized 42 studies on the exposure of bicyclists. Fourteen studies addressed BC or EC. The studies are combined and evaluated according to the reported differences between high and low exposure road segments. The exposure on high exposure segments is 8 times higher than low exposure segments across the studies when excluding rural assessments. In the measurements for the instantaneous model (reported in $\mathrm{BC}_{\text {loc,i,meas }}$ ) the high exposure locations reach levels up to 25 times the low exposure locations when including rural data and up to 10 times when excluding rural data (see Figure 5). The evaluations in the instantaneous model are aggregated by $50 \mathrm{~m}$ segments. In the review the data is aggregated by physical street segments. The measurements in the instantaneous model also include high wind speed conditions and extreme background conditions, typically not sampled in bicycle campaigns. These differences in the measurement setup (spatial detail and sampled meteorological conditions) explain the larger detected ranges. Despite the differences in the measurement setup, the measurement ranges are comparable with the results in the review. 


\subsection{Potential of background adjusted models}

The additive modeling approach has the potential to be more than a mathematical method to disentangle the meteorological and background exposure effects from the local traffic contribution. Several authors investigated the particle size distribution in relation to the distance to the source (Karner et al., 2010, Boogaard et al., 2011, Strak et al., 2011, Kingham et al., 2013, Holder et al., 2014). All results show similar trends; closer to the source the size distributions show higher numbers of small particles. In health effects of air pollution exposure research, the importance of the particle size and particle count has been investigated by different authors (Strak et al., 2010, Seaton et al., 1995). Although the smallest particles may enter the body in different ways (e.g. through the olfactory nerve (Oberdorster, 2004)), specific health effects or different toxicity estimates (based on particle mass or on particle number and size distributions) have proven hard to detect (Osunsanya et al., 2001). The additive approach -splitting exposure in background particles (aged and larger) and local contribution (fresh and small)- can be a base for adjusting the exposure to particle size corrected exposures and to verify if high local contributions result in adverse health effects. Quantifying the particle size distribution adjustments for the local and background contribution is subject to further research. Similar measurement campaigns measuring UFP particle counts or particle size distributions can add significant value in this respect. A smaller measurement campaign has already shown that this technique is also valid for UFP (Dekoninck et al., 2015). UFP shows a steeper relation to LoLF compared to BC and the additive approach is less sensitive to the background concentrations compared to BC.

\subsection{Diurnal variability, vehicle fleet composition and policy support}

The main limitation in the presented results is the restriction to rush hour exposure and traffic, the data limitation of the underlying instantaneous model. Since most policy measures are mainly focusing on commuting, the presented results are applicable in that context. The proven effectiveness of the noise sampling implies that mapping the traffic related exposure has the potential to be extended to capture the diurnal variability with only a small extension of the sampling strategy to include trips outside the rush hour and adding the hour of the day as a new dimension in the models. Only few passages on different types of routes for different times of the day could reveal the diurnal patterns of the $\mathrm{BC}$ exposure. Implementing this technique in other countries and/or continents requires recalibration of the instantaneous model. Vehicle fleet, vehicle fleet evolution, driver behavior, biking facilities, city characteristics, meteorological conditions 
and air pollution background concentration all have their specific influence on the relationship between noise and local traffic related air pollution. The sensitivity of the spatiotemporal model at different locations should be evaluated for other dependencies (temperature, humidity etc.). Validating new implementations is crucial and will not only illustrate the proposed methodology but will also detect effects of different vehicle fleets. This must be seen as one of the most important applications of the combined methods (instantaneous modeling and yearly meteorology adjusted maps). The instantaneous models will adjust and resolve the bias between the different mobile measurement campaigns for meteorology, background concentrations, route choice and traffic dynamics. The remaining difference can be attributed to the different fleet composition. In Belgium more than $60 \%$ of the vehicles use diesel, a well-known source of BC. Large changes in the relationship between noise and $\mathrm{BC}$ in other countries are probable and will be relevant for policy decisions in Belgium. Consecutive calibration measurement campaigns are relevant to quantify the changes in the vehicle fleet emission over time and require similar control over the sampling biases to reveal the vehicle fleet related component in the evolution of the $\mathrm{BC}$ exposure. The effects of changes in the local and the background exposure due to changes in the vehicle fleet emission or other BC sources are automatically included through the background adjusted approach of the instantaneous model. This leads us to an important feature of the mobile noise based traffic assessments. Noise emission of the vehicle fleet is much less sensitive to change over time due to much less restrictive and less effective legislation for the noise emission of the vehicle fleet.

412 The traffic quantification is therefore valid for longer periods of time. Only reduced combined noise-BC measurement campaigns are required to reassess the noise-BC relation. In response to local policy based traffic changes, small dedicated 'noise-only' measurements can reassess the traffic densities and traffic dynamics. The burden and costs of performing large air pollution measurement campaigns can be reduced significantly while the results cover much larger areas.

From a scientific point of view, the improvement of personal exposure assessment for bicyclists to traffic related air pollution is the most important goal of this methodological exercise. From the cyclist's point of view, the awareness of their route choice on the related exposure is the most important aspect. Only if people are aware of an issue, they can react and change their behavior accordingly. The main traffic related challenge of the local governments is reducing car use within cities and promoting a shift to other modes of 422 transportation. A campaign similar to the proposed setup can inform the public on low exposure routes for 
cyclists and the local government can use this information to improve the quality, availability and dissemination of the alternative routes. Missing links in the biking network can be detected and benefits of investing in these trajectories can be quantified and used in the dissemination process. Alternative routes and improved bicycle infrastructure will also reduce the number of bicycle accidents (Reynolds et al., 2009, Vandenbulcke et al., 2014, Molino et al., 2009).

\subsection{Multidisciplinary aspects and LURs}

The mobile noise mapping should not be only performed for air pollution assessments but can also be a part of extended multidisciplinary evaluations of in-city traffic related livability. Strong synergies exist between local air pollution exposure assessments and traffic noise related burdens. Local traffic assessment along the roads near the dwellings improves noise annoyance and wellbeing evaluations of the inhabitants (Botteldooren et al., 2010). Mobile noise measurements include more detail than calculated noise maps and have the potential to add value to all environmental noise related evaluations. They also provide a proxy for traffic on low-density roads where no external traffic data is available. An important application is the improvement of the traffic related air pollution land-use regression models. The mobile noise map provides traffic data for the LUR models on low density roads including local traffic dynamics. In the acoustic field, the combination of mobile and fixed noise monitoring stations will enable dynamic noise maps (Can et al., 2011, Can et al., 2014). City-wide mobile noise evaluation is therefore a strong tool to improve in-city livability in a multidisciplinary approach.

\section{CONCLUSIONS}

We have successfully extended an instantaneous exposure model for bicyclists to a yearly meteorology averaged exposure model. Strong seasonal effects were detected. Mapping the local variability of in-traffic exposure to $\mathrm{BC}$ for cyclists based on mobile noise measurements can be achieved with a small number of passages, since the noise exposure assessments are much less influenced by meteorological conditions and are therefore more efficient compared to in-traffic air pollution assessments. Extending the presented models to diurnal exposure models becomes feasible.

In low background conditions the background $\mathrm{BC}$ exposure accounts for less than $25 \%$ of the bicyclist's exposure, whereas in high background exposure conditions the contribution is 40 to $60 \%$. Low exposure 
route choice can reduce the local traffic exposure with at least $35-60 \%$ depending on the available alternative trajectories and the season. Local governments can use mobile noise mapping to support investments in alternative networks for cyclists.

The results support the potential of the additive approach defining personal exposure as the sum of a background contribution and a local traffic contribution. Applying this technique enables international comparison of both the local traffic related particulate matter exposure and the background exposure levels. Applying the methodology on a city-wide scale will result in a detailed spatial and accurate yearly averaged exposure map. The influence of instantaneous meteorology on air pollution exposure can be quantified through a partial participatory sensing campaign measuring one or more traffic related air pollutants for the full range of meteorological conditions in parallel to the mobile noise measurement campaign mapping the full city. Extrapolation to yearly local and total exposure with or without seasonal adjustments is inherently available in the methodology. This low cost methodology quantifies the local traffic in an unprecedented spatial resolution and fits in a multidisciplinary approach of evaluating and improving personal exposure, livability, wellbeing and health in large urban and suburban context.

Bigazzi, A. Y., \& Figliozzi, M. A. (2014). Review of Urban Bicyclists' Intake and Uptake of Traffic-Related Air Pollution. Transport Reviews, 34(2), 221-245.

Boogaard H., Kos G.P.A., Weijers E.P., Janssen N.A.H., Fischer P.H., et al. 2011. Contrast in air pollution components between major streets and background locations: Particulate matter mass, black carbon, elemental composition, nitrogen oxide and ultrafine particle number. Atmospheric Environment, 45, 650-658.

Botteldooren D., Dekoninck L. \& Gillis D. 2011. The Influence of Traffic Noise on Appreciation of the Living Quality of a Neighborhood. International Journal of Environmental Research and Public Health, 8, 777 798.

Can A., Dekoninck L. \& Botteldooren D. 2014. Measurement network for urban noise assessment: Comparison of mobile measurements and spatial interpolation approaches. Applied Acoustics, 83, 32-39.

Can A., Van Renterghem T., Rademaker M., Dauwe S., Thomas P., et al. 2011. Sampling approaches to predict urban street noise levels using fixed and temporary microphones. Journal of Environmental Monitoring, $13,2710-2719$. 
Dekoninck, L.; Botteldooren, D.; Int Panis, L., Guidelines for participatory noise sensing based on analysis of high quality mobile noise measurements. Internoise 2012 (conference), 394-402

Dekoninck L., Botteldooren D. \& Int Panis L. 2013. An instantaneous spatiotemporal model to predict a bicyclist's Black Carbon exposure based on mobile noise measurements. Atmospheric Environment, 79, 623-631.

Dekoninck L., Botteldooren D., Panis L.I., Hankey S., Jain G., et al. 2015. Applicability of a noise-based model to estimate in-traffic exposure to black carbon and particle number concentrations in different cultures. Environment International, 74, 89-98.

Dominici F., McDermott A., Zeger S.L. \& Samet J.M. 2002. On the use of generalized additive models in timeseries studies of air pollution and health. American Journal of Epidemiology, 156, 193-203.

Dons E., Panis L.I., Van Poppel M., Theunis J. \& Wets G. 2012. Personal exposure to Black Carbon in transport microenvironments. Atmospheric Environment, 55.

Holder A.L., Hagler G.S.W., Yelverton T.L.B. \& Hays M.D. 2014. On-road black carbon instrument intercomparison and aerosol characteristics by driving environment. Atmospheric Environment, 88, 183-191.

Janssen N.A.H., Hoek G., Simic-Lawson M., Fischer P., van Bree L., et al. 2011. Black Carbon as an Additional Indicator of the Adverse Health Effects of Airborne Particles Compared with $\mathrm{PM}_{10}$ and $\mathrm{PM}_{2.5}$. Environmental Health Perspectives, 119, 1691-1699.

Karner A.A., Eisinger D.S. \& Niemeier D.A. 2010. Near-Roadway Air Quality: Synthesizing the Findings from Real-World Data. Environmental Science \& Technology, 44, 5334-5344.

Li L.F., Wu J., Hudda N., Sioutas C., Fruin S.A., et al. 2013. Modeling the Concentrations of On-Road Air Pollutants in Southern California. Environmental Science \& Technology, 47, 9291-9299.

Molino J.A., Kennedy J.F., Johnson P.L., Beuse P.A., Emo A.K., et al. 2009. Pedestrian and Bicyclist Exposure to Risk Methodology for Estimation in an Urban Environment. Transportation Research Record, 145-153.

Oberdorster G., Sharp Z., Atudorei V., Elder A., Gelein R., et al. 2004. Translocation of inhaled ultrafine particles to the brain. Inhalation Toxicology, 16, 437-445.

Osunsanya T., Prescott G. \& Seaton A. 2001. Acute respiratory effects of particles: mass or number? Occupational and Environmental Medicine, 58, 154-159.

Pearce J.L., Beringer J., Nicholls N., Hyndman R.J. \& Tapper N.J. 2011. Quantifying the influence of local meteorology on air quality using generalized additive models. Atmospheric Environment, 45, 13281336.

Reynolds C.C.O., Harris M.A., Teschke K., Cripton P.A. \& Winters M. 2009. The impact of transportation infrastructure on bicycling injuries and crashes: a review of the literature. Environmental Health, 8.

Seaton A., Macnee W., Donaldson K. \& Godden D. 1995. PARTICULATE AIR-POLLUTION AND ACUTE HEALTHEFFECTS. Lancet, 345, 176-178.

Strak M., Boogaard H., Meliefste K., Oldenwening M., Zuurbier M., et al. 2010. Respiratory health effects of ultrafine and fine particle exposure in cyclists. Occupational and Environmental Medicine, 67, $118-124$. 
516 Strak M., Steenhof M., Godri K.J., Gosens I., Mudway I.S., et al. 2011. Variation in characteristics of ambient 517 particulate matter at eight locations in the Netherlands - The RAPTES project. Atmospheric 518 Environment, 45, 4442-4453.

519 Vandenbulcke G., Thomas I. \& Panis L.I. 2014. Predicting cycling accident risk in Brussels: A spatial case$520 \quad$ control approach. Accident Analysis and Prevention, 62, 341-357.

521 WHO Europe, 2012: Health effects of black carbon, ISBN: 9789289002653.

522 Wood S.N. 2006. On confidence intervals for generalized additive models based on penalized regression $523 \quad$ splines. Australian \& New Zealand Journal of Statistics, 48. 